\title{
Approche des Capabilités et sociabilité. Une étude de la vie associative des jeunes à Bamako (Mali)i.
}

\author{
Ababacar Dieng \\ Institut d'études démographiques et du parcours de vie, Université de Genève. \\ abadieng@yahoo.fr
}

\section{Résumé}

Cet article applique l'approche des Capabilités à l'analyse de la sociabilité des jeunes à Bamako. On examine l'imbrication entre la participation associative et l'entourage familial, et leurs implications en termes d'épanouissement personnel, décrit par la jouissance d'activités récréatives et de temps non structuré. Les données proviennent d'une enquête auprès de 1819 jeunes bamakois de 12 à 30 ans. Les résultats montrent que la participation associative est faible (16\%) et constitue un espace d'épanouissement variable selon le quartier de résidence, le sexe, la pratique religieuse et l'occupation. Selon les situations, la combinaison entre la participation associative et la richesse de l'entourage social produit ou gomme les inégalités. En particulier, la participation sociale, quelle que soit sa forme, ne remplit pas un rôle d'épanouissement pour les jeunes travailleurs. D'autre part, la participation associative ne modifie pas le déficit de temps non-structuré des femmes, toutefois ce handicap des femmes est effacé par un entourage riche.

Mots-clés : participation sociale, vie associative, entourage familial, temps non structuré, jeunes à Bamako, capabilités.

\section{Abstract}

\section{Capability Approach and social participation. A study of the associative life of young people in Bamako (Mali).}

In this paper we use the Capability Approach to analyse the social participation of young people in Bamako. We examine the complex relationship between the main types of social participation, i.e. associative life and family network, and their influence in terms of personal flourishing as defined by recreational activities and unstructured time. We use the data from a survey of I,819 young people aged 12 to 30 . Results show that only $16 \%$ are members of an association and that the impact of participation on personal flourishing varies with neighbourhood, sex, religiosity and type of occupation. Combining associative participation and the richness of the family network introduces or erases inequalities. In particular, regardless of its type, social participation is not a factor in personal flourishing for young people already professionally active. Associative life does not modify women's lack of unstructured time, but a rich network erases this handicap for women.

Keywords: social participation, associative life, social network, youth, Bamako, unstructured time, capabilities.

\section{Introduction}

Les profondes mutations qui touchent les sociétés africaines se manifestent également par la diversification des formes d'expression individuelles et communautaires. En effet, la modernisation de la société a fait émerger de nouveaux acteurs sociaux telles les associations et ONG. Le renforcement de la société civile semble lié aux limites des États centraux à prendre en charge nombre de problèmes rencontrés par les populations. Dans ce sens, la 
participation sociale apparalt comme une manifestation de la vie communautaire, et ceci dans une double perspective. La participation sociale est un engagement au service de la communauté par et à travers les associations et les affiliations traditionnelles ou religieuses. Elle est aussi une démarche individuelle de sociabilité dès lors qu'elle préside à l'établissement de relations sociales indépendamment des enjeux pour la communauté.

La présente recherche examine les déterminants sociaux et individuels de la sociabilité des jeunes et l'impact de leurs opportunités à cet égard sur leurs modes de vie. L'intérêt porte naturellement sur leur emploi du temps par la distinction entre les engagements individuels et ceux liés aux activités économiques et domestiques, puis entre ces obligations individuelles/collectives et le temps libre ou non-structuré. La nouvelle culture-jeune à Bamako se dessine à travers le temps non-structuré qui est un espace de liberté, d'activités diverses, de loisir, mais aussi de lieux de vie liés au périmètre familial et à l'espace communautaire et des pairs (SauvainDugerdil et Ritschard, 2005). Dans la perspective de l'approche des Capabilités, il s'agit d'établir un cadre d'analyse qui distingue les facteurs de contexte des caractéristiques socioculturelles, familiales et personnelles qui affectent les opportunités en matière d'intégration sociale et de sociabilité, mesurées ici par la participation associative et l'entourage social.

Notre première question de recherche s'attache donc à caractériser cette participation sociale et les facteurs y relatifs. On cherche à savoir pour quels jeunes l'adhésion à une association constitue une Capabilité au sens d'un espace d'opportunité en termes de sociabilité? On analyse ensuite dans quelle mesure les jeunes jouissant des mêmes opportunités, c'est-à-dire des mêmes Capabilités, se distinguent dans leurs réalisations en termes de bien-être psychologique et social. Notre seconde question de recherche porte donc sur les caractéristiques (contextuelles, de groupe, familiales et individuelles) qui font que certains jeunes participent à la vie associative dans une perspective d'épanouissement personnel plutôt que pour d'autres raisons. La troisième question examine les rôles respectifs de la participation sociale (ou associative) et des autres formes de sociabilité (l'entourage social) sur l'épanouissement personnel. On cherche à mettre en exergue les inégalités à cet égard parmi les jeunes qui jouissent du même entourage social exprimé par l'entourage familial et l'insertion sociale.

\section{Problématique et définitions:}

\section{La participation sociale comme Capabilité}

L'approche par les capabilités (AC) est un cadre théorique centré autour de concepts de base, en particulier la notion de Capabilité. Celle-ci représente la liberté, et/ou la possibilité réelle, d'être ou de faire ce que nous valorisons (Sen, 1999). Les ressources mises à disposition par le contexte sont transformées en opportunités de manière inégalitaire par les individus selon leurs caractéristiques (facteurs de conversion). Cellesci se situent à trois niveaux (individuel, familial, groupe social) et expliquent le différentiel dans la capacité à convertir les biens et ressources en des réalisations ou accomplissements (Sen, 1993 ; Robeyns, 2005 ; Bonvin et Favarque, 2008). L'accomplissement est une forme ou un aspect du bien-être individuel et social, lequel permet de démarquer les groupes vulnérables et de cibler les facteurs d'inégalité.

Avant de définir les concepts de participation sociale et de sociabilité en général, il est important de voir comment ils sont conçus par la théorie des Capabilités? Dans "Capability and Well-being ", Sen introduit une hiérarchie dans les Capabilités. II distingue d'une part les Capabilités de base liées aux accomplissements vitaux (se nourrir, boire, se loger, se soigner) pour lesquelles l'accès réel importe plus que l'éventail de choix. D'autre part, il y a les Capabilités complexes liées en particulier à l'intégration sociale des personnes, à savoir la capacité à participer à la 
vie démocratique, à la vie communautaire, être respectable et digne, etc. Ce type de Capabilités est qualifié de "complexe", car il peut comporter des contenus variables selon le contexte socioculturel. Une autre de leurs propriétés (découlant aussi de leur caractère non vital) est qu'elles impliquent une réelle liberté de choix entre plusieurs alternatives. Elles se distinguent aussi par leur dimension processuelle (Bonvin \& Farvaque, 2008). Pour justifier la relativité des Capabilités complexes qui s'oppose à l'universalité des Capabilités de bases, Sen évoque la profonde différence dans ce que les êtres humains valorisent. Davis (2009) va plus loin dans l'analyse de cette distinction et établit un lien générique entre ces deux types de Capabilitésii. Pour lui, la Capabilité basique relève d'un besoin humain qui nécessite "production " et "maintenance " (de la vie humaine), lesquelles s'inscrivent dans un processus continuel de création des structures sociales. Or, ces structures permettent le développement des Capabilités complexes notamment à travers la croissante division sociale du travail et l'hétérogénéité de l'activité humaine. L'inscription des interactions sociales ou de la sociabilité dans une dialectique rationaliste avec les besoins physiologiques s'écarte de la tradition sociologique qui en fait un objet d'étude autonome.

Selon l'usage répandu, la sociabilité d'un individu renvoie à l'aptitude, ou capacité, de celui-ci à vivre en société, tandis qu'en sociologie, la sociabilité désigne "l'ensemble des relations qu'un individu entretient avec d'autres compte tenu de la forme que prennent ces relations " (Forsé, 1991). Le glissement vers l'autonomisation du concept de sociabilité a consisté à le vider de ses connotations psychologiques: on passe de la "capacité » d'établir des contacts sociaux au simple fait de les établir (Héran, 1988). L'historique du concept de sociabilité dans les sciences sociales, et particulièrement en sociologie, montre une différenciation autant des formes que des contenus de la sociabilité selon les courants et les théories en question ${ }^{\text {iii. }}$.

Nous examinons ici la sociabilité en retenant quelques principes énoncés. Premièrement, il s'agit d'un concept autonome, c'est-à-dire étudié indépendamment des liens génériques avec les besoins physiologiques liés aux Capabilités de base. Deuxièmement, la sociabilité est à concevoir sous toutes ses formes, quel que soit le contenu de l'interaction sociale. Ce second principe permet de vider les jugements de valeur et amène à constater toutes les relations sociales qu'un individu établit, ou est en mesure de faire. II permet également de faire le lien avec la perspective des Capabilités : la sociabilité comme espace de libertés permettant aux individus de répondre au besoin d'établir des relations sociales's. Nous définissons la sociabilité comme l'ensemble des interactions ou relations sociales dont les contenus et/ou le cadre d'émergence peuvent relever de l'entourage familial, de l'insertion sociale et de la participation sociale. Dans notre schéma conceptuel de la "sociabilité », nous considérons ces trois dimensions, ou alternatives, comme étant des modes d'expression de la sociabilité individuelle.

Dans la réalité sociale, ces dimensions s'imbriquent et offrent de manière variable aux individus des opportunités de s'intégrer socialement. Pour analyser la sociabilité, il faut tenir en compte deux faits liés que nous posons comme postulats: aucune dimension n'est exclusivement nécessaire (ou obligatoire) et n'est nécessairement exclusive. Les individus peuvent «satisfaire » leur besoin de sociabilité avec l'apport des différentes dimensions, c'està-dire en combinant différents modes d'expression de la sociabilité.

La première dimension de la sociabilité, l'entourage familial, est constituée des relations d'interdépendances dans la parentèle circonscrite par les liens de sang et d'alliance. II s'agit du premier niveau d'expression de la sociabilité individuelle traduisant l'adoption et l'entretien des relations familiales dans la durée. Le poids social et biologique de la parenté 
donne une forte intensité aux relations familiales, bien qu'il soit possible d'y renoncer ou de les remettre en question.

La seconde dimension - l'insertion sociale regroupe les formes et contenus de la sociabilité relatifs aux relations développées dans l'environnement social élargi. Elle inclut les relations d'affinité (rapports amicaux ou de sympathie), les relations liées à l'occupation et aux rapports de travail (rapports entre collègues), les relations au sein du réseau élargi (réseaux de connaissances, le voisinage).

La participation sociale, troisième dimension de la sociabilité, reflète les interactions sociales établies dans le cadre de la vie communautaire avec souvent un engagement individuel vis-à-vis de la communauté. Cet engagement peut renvoyer à une quête citoyenne, à un combat politique et à un élan identitaire par le jeu d'appartenance/identification à un lieu d'origine, à une ethnie ou une religion. L'exercice de rôles et fonctions, dans un cadre organisé ou non, peut amener l'individu à établir des relations sociales avec des acteurs de l'environnement externe pour diverses raisons comme la recherche de soutien financier ou matériel, les jumelages, etc. L'exemple en est donné à Bamako, ou dans les autres villes africaines, par les jeunes qui, pour nettoyer leur quartier, démarchent d'autres acteurs susceptibles de leur apporter un soutien.

Au-delà de cette distinction en termes d'engagement communautaire, on peut s'interroger sur d'autres critères de distinction entre la participation sociale et les deux autres dimensions de la sociabilité (que l'on peut regrouper sous l'expression d'entourage social)? À cet égard, on peut formuler deux postulats théoriques. D'une part, notre schéma conceptuel de la sociabilité exprime des niveaux différenciés de densité et d'intensité des rapports interindividuels qui traduit une implication graduée de l'individu dans les rapports sociaux. Son intensité est importante au sein de l'entourage familial, moyenne pour l'insertion sociale et moindre pour la participation sociale. Donc, les rapports interindividuels semblent être caractérisés par un moindre investissement dans le cadre de la participation sociale, par rapport à celui des relations dans l'entourage social. En revanche, le critère d'élection des partenaires serait plus marqué pour la participation sociale (on choisit le groupe qu'on intègre ou quitte) que pour l'entourage familial (dans une large mesure on ne choisit pas sa famille, ses parents, ses enfants) ${ }^{\mathrm{v}}$.

Comme le souligne Zay (198I), la participation sociale est une notion plus large qui implique une action de l'individu et ne se limite pas à l'appartenance à un groupe spécifique: "La participation des personnes en tant qu'état, se concrétise par l'appartenance à des groupes formels (associations volontaires) ou non formels (cercles d'amis, voisins) et la possibilité d'en bénéficier. En tant qu'action, elle se matérialise par des rôles qu'elles peuvent jouer à l'intérieur de ces groupes... Au niveau du groupe, la participation se définit par la forme, la nature, l'intensité et la fréquence des contacts entretenus avec ceux qui en font partie... Au niveau de la société globale, la participation consiste à prendre part, d'une façon plus ou moins active, à la vie économique, politique, communautaire» (Zay, cité par Larivière, 2008, p. II7). Cette définition moins statique est plus en adéquation avec l'analyse des Capabilités, ceci pour au moins quatre raisons : a) elle donne plusieurs acceptions aux niveaux micro, méso et macro; b) elle relève les liens entre ces trois niveaux; c) la posture de l'individu est exprimée comme état et comme action ; d) différents domaines d'activisme sont possibles: récréatif, politique, citoyen, économique, communautaire.

\section{Approche méthodologique}

Données utilisées

Nous procédons ici à une analyse complémentaire, sous l'éclairage des Capabilités, des données d'une enquête par questionnaire réalisée en 2002 auprès de 1819 
jeunes de Bamako. Ce projet " chantier jeunes » du Laboratoire de démographie et d'études familiales (actuellement I-Demo) de l'Université de Genève) et de ses partenaires de l'Université de Bamako avait pour objectif d'analyser la situation des jeunes, leurs vulnérabilités, les risques et opportunités de leurs comportements. L'accent a été mis sur les biographies résidentielle et familiale, de formation et d'activités, sur les modes et conditions de vie à travers l'emploi du temps, les occupations, la santé et la santé de la reproduction et le réseau familial et social. Nous revenons dans ce volume sur ce projet qui a déjà été l'objet d'une série de publications qui a fourni une image de la transition vers l'âge adulte de jeunes africains citadins, à un moment donné et dans un contexte spécifique ${ }^{\mathrm{vi}}$, pour mieux problématiser grâce à la perspective des Capabilités la question du rôle de la participation sociale, un volet encore peu exploité.

Nous nous référons ici au module du questionnaire qui traitait de la vie associative. Les jeunes étaient invités à notifier leur appartenance à une (ou plusieurs) associations/groupements, et le cas échéant de préciser: a) Type d'association / groupement, b) Leur fonction/rôle dans cette association c) Quelles activités de cette association les intéressent le plus. Nous utilisons aussi quelques informations des modules relatifs au réseau de confidence et à l'emploi du temps. Le réseau des confidents décrit à qui les jeunes se confient en cas de problèmes dans chacun des sept domaines suivants: santé, nourriture, argent, amis, famille, tristesse, secret (voir l'analyse de Roulin et Sauvain-Dugerdil, 2009). Considérant l'emploi du temps non structuré comme un indicateur pertinent des profonds changements des modes de vie des jeunes citadins, l'enquête a inclus un module sur l'emploi du temps du samedi, la journée la moins structurée de la semaine, et a examiné ce que font les jeunes lorsqu'ils ne sont pas au travail, à l'école ou en train de faire des tâches domestiques $\mathrm{Ce}$ concept de temps non-structuré se distingue de la notion de temps libre ou de loisirs car il n'est certes pas exempt de contraintes et de normes, mais il exprime la marge d'autonomie dont jouit l'individu, cet espace de créativité qui joue un rôle fondamental dans la construction de l'identité adulte (Sauvain-Dugerdil et Ritschard, 2005 et 2009).

\section{Le schéma des Capabilités comme modèle d'analyse}

L'approche des Capabilités offre un cadre d'analyse flexible qui s'adapte au sujet et au contexte étudié. Par essence incomplet, ce cadre nécessite l'apport du chercheur pour donner forme à l'enchevêtrement logique des variables et pour choisir les outils et techniques appropriés de traitement des données. Dans ce cas précis, construire un modèle d'analyse revient à établir un schéma des Capabilités (voir les deux premiers articles de ce volume). La Figure I montre la répartition des variables entre les différents ensembles du cadre des Capabilités (facteurs de contexte, facteurs de conversion, Capabilités, réalisations). II ne représente pas un cheminement causal, mais un emboîtement de niveaux: les influences des caractéristiques du contexte sont modulées par les facteurs de conversion et les Capabilités constituent l'espace dans lequel s'inscrivent les réalisations. 
Figure I : Cartographie des variables selon le cadre des Capabilités

\section{Facteurs de contexte}

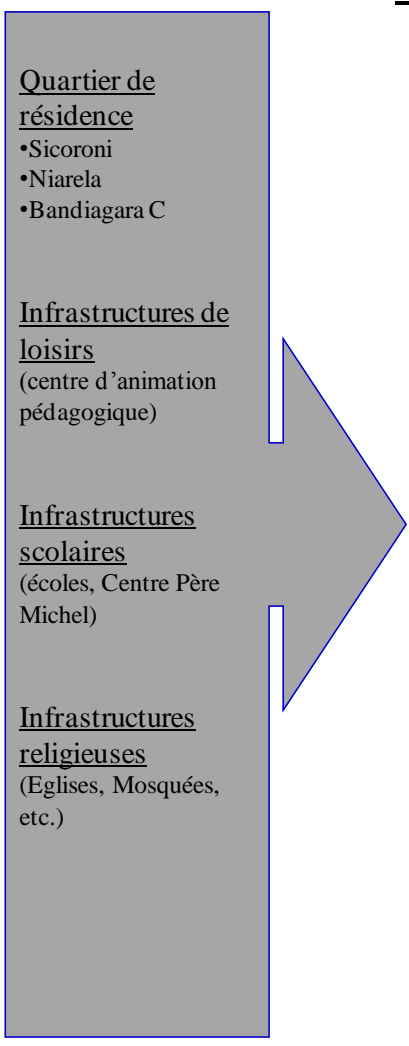

Nous considérons ici l'insertion sociale, telle que mesurée par la participation sociale et l'entourage social, comme une Capabilité. II s'agit donc de la liberté qu'ont les jeunes à mettre en pratique leur sociabilité pour atteindre un état de bien-être psychologique et social (répondant au besoin de sociabilité), à travers les choix et préférences relatifs aux types d'associations et de fonctions dans cellesci. La réalisation est conçue dans une dimension d'épanouissement personnel que nous mesurons par deux variables: l'adhésion à une association par intérêt de type récréatif et, pour l'ensemble des jeunes, la jouissance de temps non-structuré. Les réalisations s'articulent aux Capabilités à travers les choix et engagements, tels que décrits par les préférences des adhérents par rapport au type d'association et au niveau d'engagement.

Ce modèle est testé par une analyse en trois étapes. La première étape examine la
Capabilités

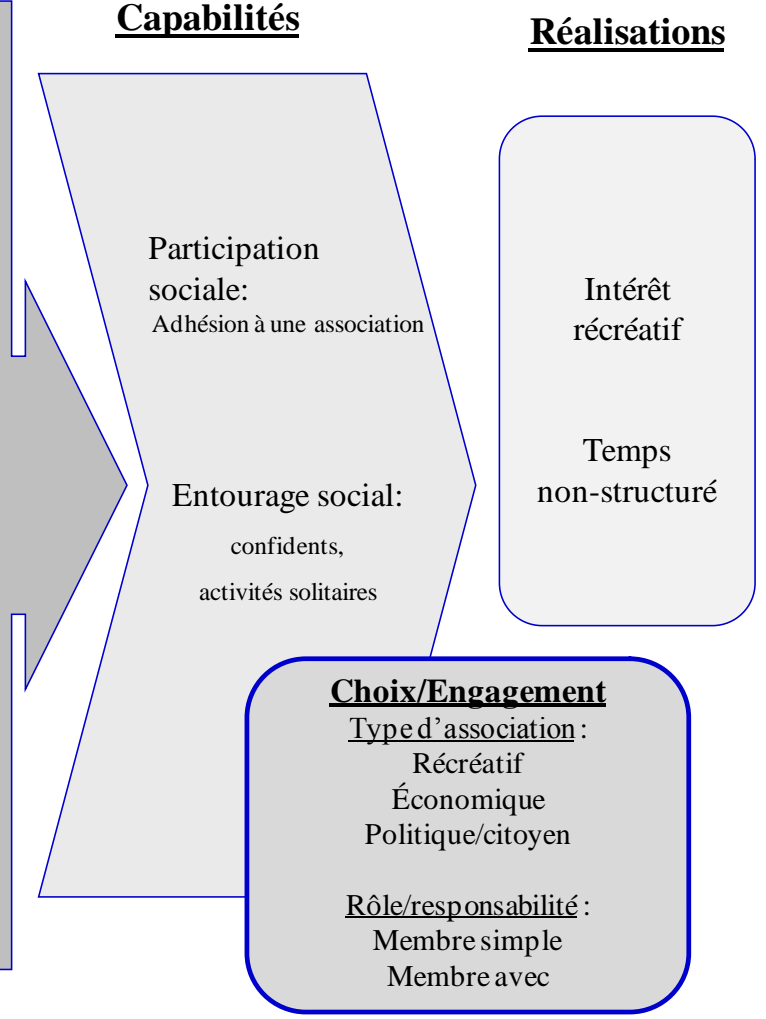

participation sociale, telle qu'exprimée par la vie associative. L'objet est la description du profil des adhérents, des types d'association, des intérêts et fonctions des membres, mais également des facteurs de l'adhésion. Dans un deuxième temps, l'analyse se focalise sur l'importance et les facteurs de variabilité de l'adhésion par intérêt essentiellement récréatif. Dans la troisième étape, on examine dans quelle mesure un entourage social riche est une alternative par rapport à la vie associative. On distingue alors les membres d'association des non-membres, et parmi ces derniers ceux avec un entourage social riche des autres. On examine les différences entre et dans les trois sous-groupes en fonction de la jouissance de temps non-structuré. Dans quelle mesure, les jeunes appartenant au même sous-groupe sont similaires à cet égard et, si tel n'est pas le cas, quels sont les autres facteurs qui expliquent ces différences. 
Description des variables (tableau I)

\section{Facteurs de contexte}

Le premier ensemble est constitué par les facteurs de contexte, à savoir les services et ressources de l'environnement local. La stratégie d'enquête a été conçue de telle manière à se centrer sur un grand quartier relativement informel dans son urbanisme et les activités de la population (Sicoroni), en le comparant d'une part à un de ses sous-secteurs (Bandiagara Coura) situé sur les collines, peuplé en majorité de migrants Dogon, et ne jouissant d'aucune infrastructure de base et, d'autre part à Niarela, un des quartiers les plus anciens de Bamako, localisé le long du fleuve Niger, aux rues pavées et doté d'infrastructures et de commerces formels nombreux. Un relevé des infrastructures disponibles (Berthé, 2006) et les résultats de l'enquête confirment que ces trois zones - Niarela, Sicoroni et Bandiagara Coura reflètent un gradient décroissant de centralité urbaine, mais aussi de dotation en infrastructures et de conditions de vie des ménages.

\section{Facteurs de conversion}

Les facteurs de conversion distribuent les variables sociodémographiques entre les trois sous-ensembles relatifs au groupe (ethnie), au ménage (caractéristiques familiales) et à l'individu (caractéristiques individuelles) :

- Ethnie: Dans les quartiers fortement touchés par l'immigration, les Bambaras, ethnie dominante dans le district de Bamako, ne représentent qu'un tiers de la population interrogée, mais ne sont que légèrement moins nombreux parmi les adhérents.

- Chef de ménage: indique le lien social entre celui-ci et l'enquêté. Plus des deux tiers des chefs sont le père et le mari, alors que très peu de jeunes sont à la tête d'un ménage.

- Type de famille: cette variable reflète la taille du ménage en décrivant sa composition. La famille restreinte, constituée des parents de premier degré, représente un tiers des cas. Elle peut concerner la famille de procréation comme celle d'orientation. La famille étendue intègre en plus les autres apparentés et les non apparentés présents dans le ménage.

- La polygamie du couple principal du ménage concerne celui des parents ou de l'enquêté lorsqu'il est marié. Les ménages polygames sont peu nombreux, mais surreprésentés parmi les adhérents.

- Le niveau de vie du ménage évalue le confort matériel du ménage. II s'agit d'un indice pondéré calculé à partir du type de construction, de son équipement et des moyens de transport.

- Sexe et âge: L'étude a interrogé pour chaque sexe trois groupes d'effectifs similaires correspondant à trois étapes de l'entrée dans l'âge adulte, une stratégie d'enquête qui a impliqué des limites d'âge différentes pour prendre en compte les spécificités de genre: adolescents (hommes: 15-19 ans; femmes: 12-14 ans), jeunes (respectivement $20-24$ et I519), jeunes adultes (25-30 et 20-25).

- Scolarité/Niveau d'instruction: cette variable est regroupée en trois modalités : I) les non-scolarisés, 2) primaire et CEP, groupe dominant (ceux ayant débuté le primaire et/ou ayant obtenu le Certificat de fin d'études primaires), 3) les diplômés de l'enmseignement fondamental (DEF) ou plus. On observe un léger gradient de l'adhésion selon le niveau de scolarisation.

- Pratique religieuse ou religiosité: tous les enquêtés étant de confession musulmane, nous considérons ici la pratique en distinguant ceux qui déclarent une pratique religieuse régulière de ceux qui pratiquent irrégulièrement ou sont non-pratiquants. Les premiers constituent près deux deuxtiers de l'échantillon,

- Statut migratoire : Déduite de la trajectoire résidentielle, cette variable donne la durée de résidence à Bamako en trois modalités. 
On constate qu'un-tiers a passé 10 ans ou moins à la capitale.

- Occupation: indique si, au moment de l'enquête, la personne interrogée exerce une activité économique, est en formation ou est sans activité économique (par exemple femme au foyer).

Tableau I : Description des variables: effectifs et taux d'adhésion

\begin{tabular}{|c|c|c|c|}
\hline Variables & Modalités & Effectif total en \% & \begin{tabular}{|l} 
Effectif $\quad$ total \\
d'adhérents en $\%$
\end{tabular} \\
\hline \multicolumn{2}{|l|}{ Effectifs absolus } & 1819 & 297 \\
\hline \multirow{3}{*}{ Zones de résidence } & Sicoroni & 66,1 & 14,2 \\
\hline & Bandiagara Coura & 17,4 & 21,8 \\
\hline & Niarela & 16,5 & 19 \\
\hline \multirow{2}{*}{ Ethnie } & Bambara & 39,6 & 14,4 \\
\hline & Autres ethnies & 60,4 & 17,6 \\
\hline \multirow{3}{*}{ Chef de ménage } & Père & 54,5 & 15,6 \\
\hline & Mari & 13,7 & 18,9 \\
\hline & Autre & 31.8 & 16.4 \\
\hline \multirow{2}{*}{ Type de famille } & Familles étendues & 65,4 & 16.7 \\
\hline & Familles restreintes & 34,6 & 15.7 \\
\hline \multirow{2}{*}{ Polygamie de égo ou ses ascendants } & Famille non polygame & 91 & 16,3 \\
\hline & Famille polygame & 9 & 17,1 \\
\hline \multirow{3}{*}{ Niveau de vie du ménage } & Faible & 36,4 & 16,5 \\
\hline & Moyen & 63,8 & 16,7 \\
\hline & Élevé & 36,2 & 15,9 \\
\hline \multirow{2}{*}{ Sexe } & Hommes & 48,2 & 15,3 \\
\hline & Femmes & 51,8 & 17,3 \\
\hline \multirow{3}{*}{ Age } & Adolescents & 33,6 & 11,3 \\
\hline & Jeunes & 34,1 & 19,7 \\
\hline & Jeunes adultes & 32,3 & 18 \\
\hline \multirow{3}{*}{ Niveau de scolarisation } & Non scolarisés & 35,6 & 14,2 \\
\hline & Primaire et CEP & 46,5 & 16,7 \\
\hline & DEF et plus & 17,9 & 19,9 \\
\hline \multirow{2}{*}{ Statut matrimonial } & Non marié (célibataire, veuf, divorcé) & 79,8 & 15,7 \\
\hline & Marié & 20,2 & 18,8 \\
\hline \multirow{2}{*}{ pratique religieuse } & Non/irrégulière & 39,1 & 15,6 \\
\hline & Régulière & 60,9 & 17,3 \\
\hline \multirow{3}{*}{$\begin{array}{l}\text { Statut migratoire (durée de résidence à } \\
\text { Bamako) }\end{array}$} & $0-10$ ans & 33,8 & 17,1 \\
\hline & II -20 ans & 47,6 & 15,8 \\
\hline & $21-30$ ans & 18,6 & 16 \\
\hline \multirow{3}{*}{ Occupation } & Actif rémunéré & 37,1 & 14,3 \\
\hline & En formation & 44 & 17,8 \\
\hline & Sans activité rémunérée & 18,9 & 17,3 \\
\hline \multirow{2}{*}{ Réseau de confidence (dans 7 domaines) } & confident dans 2 à 7 domaines & 65,3 & 15,5 \\
\hline & confident dans I domaine au plus & 34,7 & 17,9 \\
\hline \multirow{2}{*}{ Temps partagé avec l'entourage } & Solo 0 à 5 heures & 47,7 & 15,6 \\
\hline & Solo 6 heures ou plus & 52,3 & 17 \\
\hline \multirow{3}{*}{ Type d'association } & Association économique & 43,1 & \\
\hline & Association récréative & 35,7 & \\
\hline & Association politique/religieuse & 21,2 & \\
\hline \multirow{2}{*}{ Rôle } & Membre avec rôle & 46 & \\
\hline & Membre simple & 54 & \\
\hline
\end{tabular}




\begin{tabular}{|l|l|l|l|l|}
\hline Type d'intérêt & intérêt récréatif & 52,5 & \\
& & Intérêt économique & 16,5 \\
& & Intérêt politique/religieux/civique & 27,3 & \\
\hline Temps non-structuré $\quad$ (journée du & $\begin{array}{l}\text { Moins } 1 / 2 \text { journée } \\
\text { samedi) }\end{array}$ & $1 / 2$ journée et plus & 43,6 & 15,8 \\
\hline
\end{tabular}

\section{Les Capabilités}

Nous avons deux Capabilités alternatives avec la participation sociale et l'entourage social. La participation sociale est mesurée par le taux d'adhésion et l'entourage social est considéré à partir de deux variables :

- Le réseau de confidents qui distingue les jeunes ayant un réseau riche, à savoir ayant déclaré avoir un confident dans au moins deux des 7 domaines considérés (deuxtiers des jeunes), de ceux ayant un réseau pauvre, c'est-à-dire qui n'ont de confident que dans un seul domaine ou qui n'en ont pas du tout.

- Le temps passé en solo qui sépare les jeunes en deux groupes d'effectifs similaires: ceux qui ont été seuls moins de 6 heures des solitaires pour 6 heures et plus.

L'indicateur de sociabilité retenu conjugue ces deux variables pour distinguer un entourage social pauvre (6 heures ou plus en solo, et sans confident ou dans un seul domaine), un entourage social riche (moins de 5 heures passées seul et avec confidents dans au moins 2 domaines).

\section{Les choix et réalisations}

Les associations recensées ont été classées en trois groupes. Par ordre d'importance, il s'agit des associations de type économiques, récréatives et politiques/religieuses. Près de la moitié des jeunes adhérents déclarent avoir une responsabilité dans une association. Nous allons examiner plus loin l'éventuelle influence de ces types d'engagements sur la réalisation telle qu'exprimée par une dimension récréative de l'adhésion, laquelle constitue la motivation principale de la moitié des adhérents, alors qu'un peu plus d'un quart le fait par engagement communautaire et moins encore (16\%) par intérêt économique.

En parallèle, la jouissance de temps nonstructuré est une réalisation modulée par la participation sociale (adhésion) et l'entourage social. Le temps non structuré, regroupe les occupations variées qui n'entrent pas dans les activités professionnelles, les études et les tâches domestiques. II s'agit ici du temps consacré respectivement au repos, à regarder la télévision, à " faire la fête ", à faire du sport, aux causeries ou autres (notamment visite aux parents). Nous n'opérons donc pas de distinction entre les activités sociales et nonsociales, actives ou passives.

\section{Résultats}

Le profil des adhérents

Le taux de participation associative est particulièrement faible, seuls $16,3 \%$ des jeunes interrogés déclarent être membre d'une association.

Ces adhérents présentent-ils un profil particulier ? L'analyse des différences en matière d'adhésion (régression logistique) montre que ce sont les zones de résidence et l'appartenance ethnique qui jouent un rôle important (Tableau 2). 
Tableau 2: Les facteurs d'adhésion à une association.

Modèles de régression logistique sur la probabilité d'adhérer à une association.

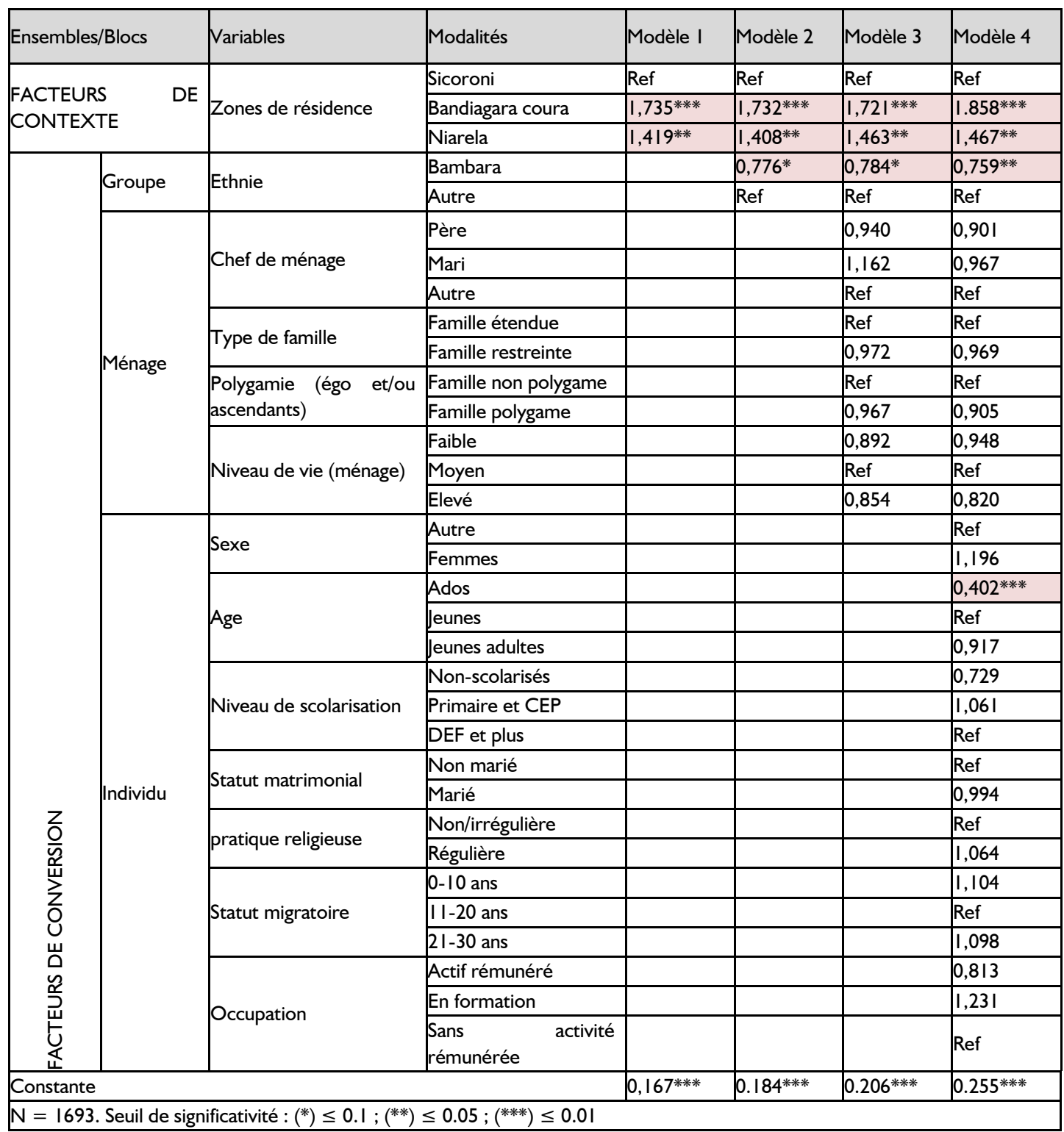

Même en contrôlant par les caractéristiques familiales et individuelles, le lieu de résidence influence de façon très significative la participation associative: les jeunes du grand quartier de Sicoroni ont une vie associative beaucoup moins marquée. Ainsi, son mode de vie plus lié à l'informel que Niarela semble se refléter aussi en termes de regroupement social et associatif. La différence est encore plus grande entre Sicoroni et Bandiagara Coura qui est certes moins loti en infrastructures, mais plus structuré par son homogénéité spatiale et culturelle. On constate que la vie associative est également plus marquée parmi les jeunes n'appartenant pas à l'ethnie dominante à Bamako, les Bambara. L'influence de l'appartenance ethnique est encore plus marquée lorsque l'on contrôle pour les caractéristiques personnelles. Ainsi, l'appartenance à une minorité ethnique favorise 
le reflexe grégaire tel qu'exprimé par la participation associative.

Les caractéristiques familiales ne jouent aucun rôle : vivre dans un ménage plus grand et élargi n'influence pas la propension des jeunes à adhérer à une association. Elle serait légèrement moindre dans les ménages plus aisés, mais la différence de probabilité n'atteint pas le seuil de significativité statistique.

En termes de caractéristiques individuelles, les non scolarisés et les jeunes économiquement actifs auraient une vie associative moins marquée, mais la différence n'est pas significative. D'un autre côté, les plus jeunes de l'échantillon sont moins participatifs, leur probabilité d'adhésion n'est que la moitié de celle des deux groupes plus âgés.
Types d'association et d'intérêts

Les adhérents appartiennent à une association et une seule. Faut-il en déduire que le type d'association reflète la motivation et l'intérêt de l'adhérent?

On peut observer dans la Figure 2 que l'intérêt récréatif est une motivation importante quel que soit le type d'association (d'importance similaire à l'intérêt politique dans les associations politiques et religieuses). Les associations récréatives enregistrent peu d'intérêt économique, celles économiques peu d'intérêt politique et enfin celles politiques quasiment pas d'intérêt économique. La relation entre le type d'intérêt et le type d'association est statistiquement significative (Phi $=0.485^{* * *} ; V$ de Cramer $\left.=0.343^{* * *}\right)$. Toutefois, le type d'association, tout comme le type d'intérêt sont aussi liés à d'autres variables (résultats non reproduits).

Figure 2 : L'intérêt des adhérents selon le type d'association

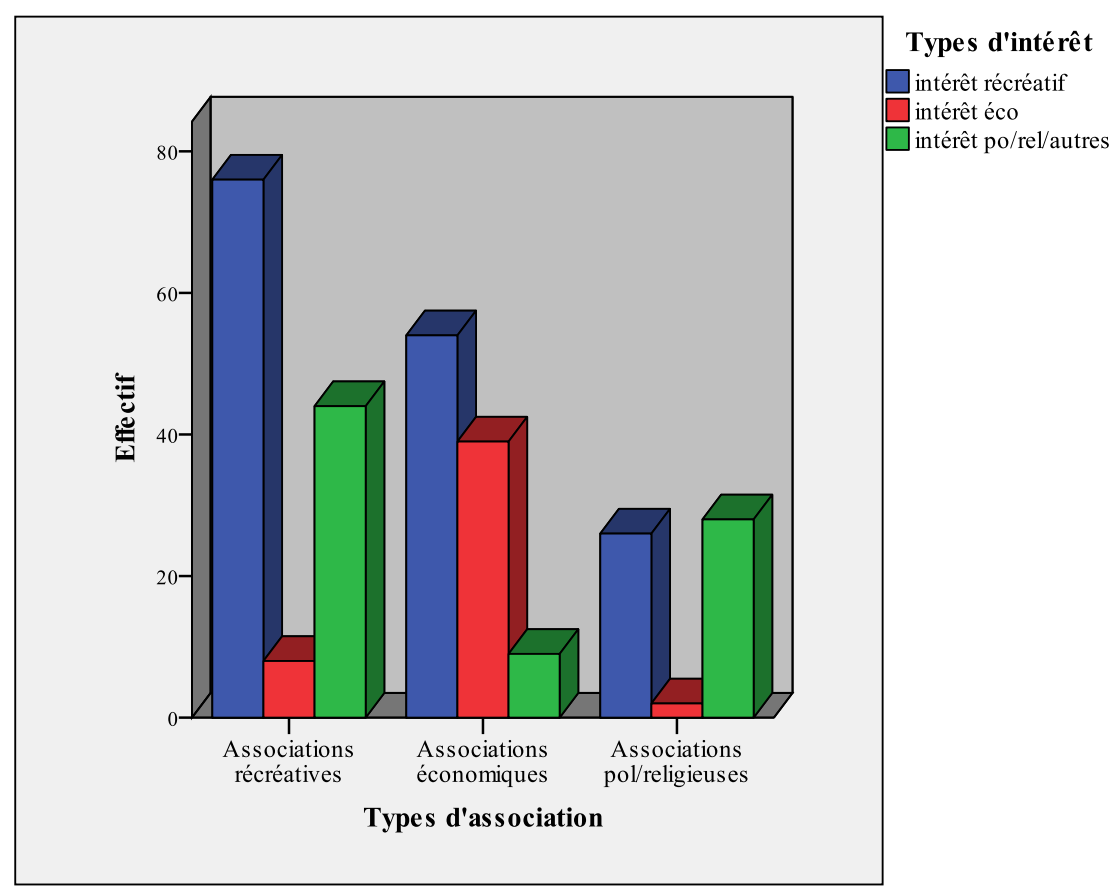

Le type d'association auquel on adhère est l'occupation. Les données (non figurées ici) fonction entre autres du quartier, du sexe et de indiquent que les jeunes de Sicoroni adhèrent 
plutôt aux associations économiques et un peu moins à celles politiques et religieuses comparativement à Niarela, quartier où le standard de vie est le plus élevé. Les femmes rechercheraient plus les associations de type économique et les jeunes encore aux études les associations de type récréatif. Par contre, la pratique religieuse ne conditionne pas l'adhésion à un type particulier d'association.

L'intérêt récréatif n'apparaît pas influencé par les caractéristiques du ménage, ni par celles du groupe social d'appartenance. La relation entre le type d'association et le type d'intérêt est confirmée par l'effet significatif du type d'association sur les autres facteurs qui influencent l'intérêt récréatif. En effet, le contrôle par le type d'association concernant l'intérêt récréatif double la différence de genre, rend significative celle selon l'occupation et réduit l'effet de la religiosité. En d'autres termes, il y a un effet genre dans le choix du type d'association auquel on adhère, cependant les femmes ont la particularité de poursuivre leur intérêt récréatif le plus souvent dans les associations économiques. Contrairement aux hommes, qui s'intéressent peu aux associations économiques, elles montrent ainsi leur dynamisme tout en alliant l'utile à l'agréable. II est intéressant de relever l'effet déterminant du type d'association par rapport à l'occupation et qui semble indiquer que malgré leur adhésion dans des associations récréatives, les jeunes en formation n'y recherchent pas pour autant les loisirs.

La religiosité ne conditionne pas le choix du type d'association, d'où l'effet neutralisant de ce dernier dans la recherche de l'intérêt ludique.

La richesse de l'entourage social comme alternative à la vie associative

Jusque-là, les analyses effectuées ont porté sur les facteurs liés à la vie associative et les caractéristiques des individus qui adhèrent dans un but de réalisation personnelle, à savoir par intérêt en termes ludiques. Nous nous interrogeons finalement sur la complémentarité entre la vie associative et la qualité de l'entourage social eu égard à l'épanouissement tel qu'exprimé dans la jouissance de temps nonstructuré. Est-ce que le taux de participation associative spécialement faible peut être expliqué par d'autres types de sociabilité en particulier la qualité de l'entourage social?

L'entourage social, mesuré par le temps en solo et le nombre de confidents, renvoie aux deux premières dimensions du concept de sociabilité à savoir l'entourage familial et l'insertion sociale. Sur le plan analytique, considérer l'entourage social comme alternative à la participation associative revient à examiner une association -supposée négative - entre eux. Or, la mesure d'association entre la vie associative et la qualité de l'entourage ne montre pas de différences significatives dans la répartition de l'échantillon.

On peut alors se poser la question de savoir si ces deux types de sociabilité ont un effet sur la qualité de vie - telle que mesurée par la jouissance de temps non structuré - et le différentiel sur ce plan. Selon les résultats, ces deux types semblent se distinguer. Nous avons vu plus haut que le temps non-structuré se distribue de façon très similaire selon l'adhésion ou non à une association (Tableau I); en revanche, il apparaît associé à la richesse de l'entourage social (Tableau 3): plus de deux tiers des jeunes qui ont un "entourage social riche " déclarent passer plus de la moitié de la journée du samedi en temps non-structuré, alors que cette proportion descend à près d'un demi pour ceux qui ont un "entourage social pauvre ». 
Tableau 3 : Mesure d'association entre l'importance du temps non-structuré et l'indicateur de sociabilité

\begin{tabular}{|c|c|c|c|c|}
\hline & & \multicolumn{3}{|l|}{ Entourage social } \\
\hline & & $\begin{array}{l}\text { Entourage social } \\
\text { riche }\end{array}$ & $\begin{array}{l}\text { entourage social } \\
\text { pauvre }\end{array}$ & Total \\
\hline \multirow{6}{*}{$\begin{array}{l}\text { Temps non-structuré } \\
\text { (journée du samedi) }\end{array}$} & \multirow{2}{*}{ Moins de la moitié journée } & 283 & 509 & 792 \\
\hline & & $31.6 \%$ & $55.1 \%$ & $43.6 \%$ \\
\hline & \multirow{2}{*}{ Moitié et plus de la journée } & 612 & 414 & 1026 \\
\hline & & $68.4 \%$ & $44.9 \%$ & $56.4 \%$ \\
\hline & \multirow{2}{*}{ Total } & 895 & 923 & 1818 \\
\hline & & $100.0 \%$ & $100.0 \%$ & $100.0 \%$ \\
\hline
\end{tabular}

On constate que l'adhésion à une association

Des régressions logistiques sur la probabilité de consacrer plus de la moitié de la journée du samedi au temps non-structuré nous permettent de voir les inégalités à l'intérieur de chacun des quatre sous-groupes vivant la même situation de sociabilité (Tableau 4).

On compare ainsi les membres d'une association, par rapport aux non membres, puis on distingue parmi ces derniers les jeunes qui ont un entourage social paurre de ceux qui ont un entourage social riche. Une des questions alors qui se pose est de savoir si la participation associative gomme les inégalités liées au temps non-structuré. efface les inégalités liées au contexte de résidence: les jeunes résidant dans la zone la plus périphérique, Bandiagra Coura, sont nettement défavorisés parmi les non membres, mais ne se distinguent pas parmi les membres. On peut faire la même observation chez des jeunes déjà actifs professionnellement : la vie associative corrige leur handicap par rapport aux non-membres. Chez les non-membres, le handicap subsiste aussi quel que soit la richesse de l'entourage. Autrement dit, les jeunes actifs n'atteignent pas la demi-journée en temps non-structuré même s'ils disposent d'un entourage social riche. 
Tableau 4: Modèles de régression logistique sur le temps non-structuré dans les quatre sous-groupes.

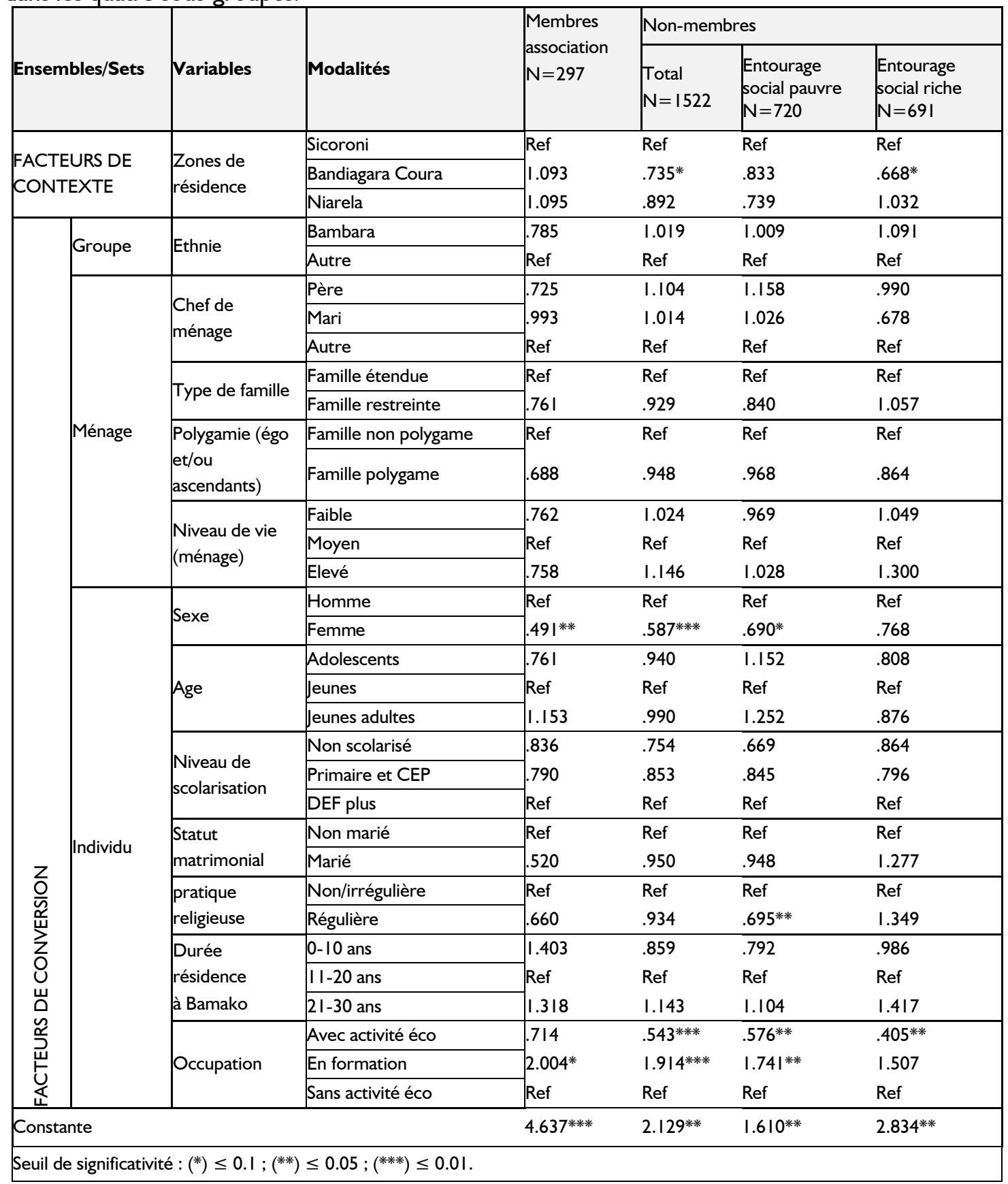


On ne note pas de différences du fait de l'adhésion pour les femmes et les jeunes en formation. L'adhésion à une association ne gomme pas le désavantage des femmes, par contre la richesse de l'entourage social chez les non-adhérentes leur permet de rattraper les hommes sur ce plan. Chez les adhérents comme les non-membres, les jeunes en formation ont deux fois la probabilité d'avoir une demi-journée de temps non-structuré comparativement aux non-actifs.

Les différences - plutôt surprenantes notées chez les jeunes en formation se retrouvent au niveau de la qualité de l'entourage social: ceux avec un entourage social pauvre jouissent plus du temps de loisir que ceux bénéficiant d'un entourage social riche.

Pour les jeunes non membres résidant à Bandiagara Coura, c'est au contraire lorsque l'entourage est plus riche qu'ils sont désavantagés à cet égard. On peut émettre alors l'hypothèse que, dans cette zone plus homogène culturellement, un réseau plus actif peut impliquer des obligations collectives, que l'on n'assume pas si l'on est membre d'une association.

En revanche, parmi les non membres, la richesse de l'entourage social estompe les inégalités liées à la pratique religieuse. En effet, lorsqu'ils ont un entourage social pauvre les jeunes pratiquants réguliers jouissent de moins de temps non-structuré, mais leur handicap disparaît parmi ceux qui bénéficient d'un entourage riche, c'est-à-dire qui sont rarement seul ou sans confident.

\section{Conclusion}

Nous avons pu appliquer l'approche des Capabilités bien que l'enquête n'ait pas été conçue selon cette perspective, parce qu'elle propose un cadre logique d'analyse qui n'est pas construit une fois pour toutes, mais est modulable en fonction du contexte et des données à disposition. Nous considérons ici la participation sociale, telle que décrite par l'adhésion à une association et la richesse de l'entourage, comme une Capabilité qui ouvre des opportunités d'épanouissement personnel.

L'analyse montre que la participation associative des jeunes à Bamako est particulièrement faible (16\%) et modulée par les facteurs de contexte, les caractéristiques liées à l'appartenance ethnique et à l'âge : résider à Sicoroni, être bambara et adolescent constituent des blocages à l'adhésion. Les jeunes de Sicoroni sont moins souvent membres d'association et leur adhésion ne relève guère de l'épanouissement personnel. Cependant lorsqu'ils ne sont pas membres, ils jouissent de plus de temps non-structuré le samedi que ceux de Bandiagara Coura. On peut alors penser que les jeunes de Sicoroni, quartier à l'urbanisme informel, moins équipé en infrastructures et avec une population culturellement hétérogène ont une sociabilité moins formalisée que les deux autres quartiers. Les jeunes de Bandiagara Coura semblent avoir une sociabilité plus structurée du fait de la dimension socioculturelle que partagent les résidents qui proviennent généralement de la récente migration - plus exactement de l'exode rural- des Dogons.

En considérant l'adhésion comme Capabilité pouvant ouvrir un espace d'opportunité, un deuxième objectif a été de voir quelles caractéristiques liées aux individus, aux ménages, au groupe social et au contexte favorisent l'intérêt de type récréatif, considéré comme réalisation personnelle. L'intérêt poursuivi, au-delà de la simple adhésion à une association, relève d'une motivation et d'un engagement personnels, c'est donc logiquement que les caractéristiques du contexte (la zone de résidence), celles individuelles (le sexe, la pratique religieuse, l'occupation) et le type d'association ont une influence significative. L'intérêt recherché par les adhérents reste logiquement, mais faiblement, associé au type d'association. L'intérêt récréatif est fortement présent dans tous les types d'associations. La particularité des femmes est qu'elles recherchent plus un intérêt récréatif bien 
qu'elles adhèrent en majorité dans des associations de type économique. A l'opposé, les hommes sont plus souvent membres d'associations récréatives et politiques/religieuses

qu'économiques. L'adhésion des femmes aux associations de type économique s'expliquerait notamment par certaines pesanteurs sociales qui rendent encore difficile leur accès au monde travail, mais la participation associative apparaît aussi comme une formidable manière d'allier l'utile à l'agréable.

La pratique religieuse régulière amoindrit la recherche de l'intérêt ludique. En revanche, il est surprenant de noter que les adhérents encore en formation jouissent moins de l'intérêt récréatif que les non-actifs et actifs, cela en dépit du fait qu'ils sont principalement membres d'associations à but récréatif.

La démarche a également consisté à considérer l'effet de la participation sociale sur la jouissance de temps non-structuré comme indicateur de bien-être socio-psychologique et, à cet égard, le rôle que pourrait jouer l'entourage social comme alternative à la participation associative. L'analyse par sousgroupe a permis de comparer les membres avec les non-membres et, chez ces derniers, ceux avec un entourage social riche par rapport à ceux ayant un entourage social pauvre. On observe que la participation associative gomme le handicap, ou corrige les inégalités, de jouissance de temps non structuré pour les adhérents qui résident à Bandiagara Coura, le quartier peu doté en infrastructures, ainsi que pour les jeunes professionnellement actifs. Parmi ces derniers, le handicap des nonadhérents persiste même lorsqu'ils disposent d'un entourage social riche, ainsi la participation sociale quelle que soit sa forme ne remplit pas un rôle d'épanouissement personnel pour les jeunes travailleurs. D'autre part, la participation associative ne modifie pas le déficit de temps non-structuré dont souffre les femmes par rapport aux hommes, ni l'avantage à cet égard des jeunes encore aux études. Parmi les nonmembres, des différences importantes sont également notées entre les jeunes selon la richesse de leur entourage. Un entourage social riche annule le handicap des femmes, des jeunes en formation et des pratiquants réguliers, leur entourage leur fournirait donc des appuis les libérant de certaines tâches. En revanche, ce n'est pas le cas des résidents de Bandiagara Coura pour lesquels, au contraire, un entourage riche pourrait impliquer des tâches accrues.

En conclusion, le premier constat est que ni l'adhésion à une association, ni un entourage social riche semblent diminuer la jouissance de temps non-structuré, pour le second c'est même le contraire: les jeunes ayant un entourage social plus riche sont aussi ceux qui ont un peu plus de temps. Si, globalement, la participation sociale n'entre donc pas en concurrence avec l'épanouissement personnel, ce n'est toutefois pas le cas pour certains jeunes qui apparemment ne peuvent pas concilier les deux, tels ceux qui résident à Bandiagara Coura, qui sont plus souvent membres d'association, mais jouissent de moins de temps non structuré, que les jeunes des deux autres zones considérées. Pour eux, la participation sociale ne constituerait donc pas une opportunité pour leur épanouissement, à savoir une Capabilité. Dans certaines situations, au contraire, la participation sociale peut atténuer un handicap. C'est ainsi que la participation associative semble diminuer le désavantage en termes de jouissance de temps non structuré des jeunes actifs professionnellement et que l'un entourage riche diminue le manque de temps des femmes et des plus religieux.

\section{References}

Berthé, Fatou (2006), Expérience du projet "Chantier Jeunes " en matière d'analyse des ressources et stratégies de la jeunesse face au défi de l'incertitude. Rapport de stage. Institut Supérieur des Sciences de la Population, Université de Ouagadougou.

Bonvin, Jean-Michel et Nicolas Farvaque (2008), Amartya Sen, Une politique de la liberté, Paris, Michalon. 
Davis, John B. (2009), The capabilities conception of the individual, Review of Social Economy, 67 (4), 413-429.

Forsé, Michel (1991), Les réseaux de sociabilité : un état des lieux, L'Année sociologique, 4I, 247-264.

Héran, François (1988), La sociabilité, une pratique culturelle, Economie et statistique, 216, 3-22.

Larivière, Nadine (2008), Analyse du concept de la participation sociale : définitions, cas d'illustration, dimensions de l'activité et indicateurs, Canadian Journal of Occupational Therapy, 75 ( 2), II4-I27(14).

Pelletier, L. G., Vallerand R. J., Green-Demers I., Bière N. M. et Blais M. R. (1995), Loisirs et bien-être psychologique, Revue Canadienne des sciences du comportement, 27(2), I40-I56.

Platt, Lucinda (2006), Social Participation: How does it vary with illness, caring and ethnic group? ISER Working Paper I8. Colchester, University of Essex.

Quéré, Louis (1988), Sociabilité et interactions sociales. Réseaux, 6(29), 75-9I.

Rivière, Carole Anne (2004), La spécificité française de la construction sociologique du concept de sociabilité, Réseaux, I(I23), 207231.

Robeyns, Ingrid (2005), The Capability Approach: a theoretical survey, Journal of Human Development, 6(I), 93-II4.

Roulin, Emily et Claudine Sauvain-Dugerdil (2009), L'espace de fonctionnement comme indice des transformations des modes de vie des jeunes malien(-enne)s. UIESP, XXVlè Congrès International de la Population, Marrakech.

Sauvain-Dugerdil, Claudine et Gilbert Ritschard (2005), Temps non structuré et espaces de vie : risques ou opportunités d'une nouvelle liberté ? Enquête auprès de jeunes maliens. UIESP, XXVè Congrès International de la Population, Tours (France).

Sauvain-Dugerdil, Claudine et Gilbert Ritschard (2009), Un samedi à Bamako. L'émergence d'une nouvelle culture-jeunes à travers
I'utilisation du temps non structuré, Ouvrage en hommage à Thérèse Locoh (J. Vallin, ed), INED Paris.

Sen, Amartya (1993), Capability and Well-Being, in M. Nussbaum and A. Sen (eds) The Quality of Life, Oxford: Clarendon Press, 30-53.

Sen, Amartya (1999), Development as Freedom, Oxford: Oxford University Press.

Simmel, Georg (1999), Sociologie. Etudes sur les formes de la socialisation, Paris, PUF, collection "Sociologies", (première édition allemande 1908).

Zay, N. (198I), Dictionnaire de gérontologie sociale, Les Presses de l'Université de Laval.

\footnotetext{
' Une version antérieure de ce travail a été publiée dans les Actes en ligne du Colloque AIDELF, Ouagadougou 2012 (à paraître).
}

ii L'auteur précise: " people are constantly involved in developing both kinds: their complex capabilities in order to further pursue their interests and develop their skills and their basic capabilities in order to maintain the skills that ensure their survival in the changing circumstances of life » (Davis, 2009, p.422).

iii Carole Anne Rivière (2004) retrace I'historique de ce concept dans la tradition française en comparaison avec les autres courants malgré leurs inter-influences. La tradition allemande a vu l'émergence de la notion de sociabilité avec les travaux de G. Simmel. Ce dernier voit dans l'interaction sociale une " réalité sociale pure ", c'est-à-dire dépourvue de finalité rationnelle utilitaire. Le déterminisme de la tradition française amène Gurvitch à envisager la sociabilité hors des rapports interpersonnels. Gurvitch distingue deux formes de sociabilité : le Nous (les intuitions collectives d'appartenance et d'identité) qui existe sans interactions sociales, le Rapport à autrui qui est la manifestation de la sociabilité à travers les rapports interpersonnels. La tradition américaine s'inspire de ces deux perspectives en considérant d'une part le rapport interindividuel à la base du regroupement social, d'autre part la forme structurante du regroupement social aussi important que le rapport individuel (Rivière, 2004). L'analyse des réseaux sociaux est née de cette tradition américaine. Il est également intéressant de voir l'article de Louis Quéré (1988) sur la sociologie formelle des interactions et relations sociales. II retrace l'histoire de la théorie de la société basée sur les relations sociales en allant de Simmel à l'ethnométhodologie.

iv On admet en sciences sociales que la sociabilité soit conçue comme un besoin à satisfaire. Pour Simmel, la sociabilité a la particularité d'être un besoin dont la satisfaction pour un individu n'entre pas en concurrence avec celles des autres individus : "Chacun devrait obtenir autant de satisfaction de ce besoin (de sociabilité) qu'il est possible de concilier avec celle de tous les autres (. . .) La joie de l'individu particulier est absolument liée au fait que les autres soient également à leur 
aise. Dans ce cas, personne ne saurait éprouver de satisfaction au prix de sentiments entièrement contraires à ceux de l'autre » (Simmel, 1981, pp. 127-128).

v Catherine Paradeise propose la définition de la sociabilité privée comme " l'ensemble des activités dont l'exercice suppose la libre élection des partenaires. " (Rivière, 2004, p. 220). Il est aussi légitime, comme le relève Rivière, de se poser la question de savoir s'il y a effectivement libre élection des partenaires dans la participation aux associations.

vi Voir aussi chapitre 4 qui revient sur le volet santé de cette même enquête et nous renvoyons le lecteur à ce chapitre pour une description plus détaillée de l'enquête. 